Optimal Evasion Against a Proportionally Guided Pursuer

by

Joseph Z. Ben-Asher

Thesis submitted to the Faculty of the

Virginia Polytechnic Institute and State University

in partial fulfillment of the requirements for the degree of

Master of Science

in

Acrospace and Ocean Engineering

APPROVED:

Eugene M: Cliff

Henry J. Kelley

Frederick I. Latze

September, 1986

Blacksburg, Virginia 


\title{
Optimal Evasion Against a Proportionally Guided Pursuer
}

by

\author{
Joseph Z. Ben-Asher \\ Eugene M. Cliff \\ Aerospace and Ocean Enginecring \\ (ABSTRACT)
}

We consider the problem of optimal evasion when the pursuer is known to employ fixed gain proportional navigation. The performance index is a measure of closest approach. The analysis is done for planar motions at constant speed. The kinematics are first linearized around a nominal collision course. The dynamics of the opponents are modeled by first order systems and their accelerations may be bounded.

Three cases are studied : unconstrained optimal evasion (where the evader is not subjected to any path constraint ) against a single pursuer, optimal evasion with a terminal path angle constraint for the evader and optimal evasion against more than one pursucr.

The optimal controls are shown to be 'bang - bang' with the number of switches depending on the pursuer's navigation gain and on the particular constraints of each case. 


\section{Acknowledgements}

I am indepted to Dr. Eugene Cliff for his help and guidance in furthering this work. I also wish to thank Dr. H. J. Kelley and Dr. F. Lutze for serving on my committee. I would like to thank the Israel Military Industries for helping and encouraging $\mathrm{me}$ in my Graduate studies. 


\section{Table of Contents}

1. Introduction 1

1.1 Historical Background 1

1.2 Objectives and Motivation 3

2. Optimal Evasion Against a Single Pursuer 5

2.1 Basic Assumptions and Equations 5

2.2 Problem Formulation $\quad 7$

2.3 Problem Analysis 9

2.4 Computational Results 11

3. Optimal Evasion With a Path Angle Constraint 13

3.1 Problem Formulation 13

3.2 Problem Analysis 15

3.3 Computational results and Problem Decomposition 19 
4. Optimal Evasion Against Two (or more) Pursuers

4.1 Problem Formulation

4.2 Problem Analysis

4.4 Computational Results

5. Conclusions 30 


\section{Table of Illustrations}


9. Switching Points versus Terminal Path Angle for $t_{f}=10 \tau_{p}$

10.Miss Distance Influence Function

11.Payoff versus Constraint

12.Payoff versus Nominal Time Between Impacts

13.Expected Payoff versus Terminal Uncertainty 


\section{Introduction}

\subsection{Historical Background}

Pursuit-evasion problems have been traditionally classified among the classical examples of differential game theory. In the last 15 years a different approach has been applied to these problems, namely, to fix the pursuer's stratcgy and to form a one -sided optimal control problem for the evader. This approach, being conceptually simpler than the former, enables more realistic models to be applied for the dynamics of the opponents. In general the fixed pursuer's strategy has been taken as constant gain proportional navigation which, under some formulations, is an optimal strategy for the pursuer.

The problem, so formulated, was solved by Julich and Borg [2] who applied a two-dimensional non-linear model and used a direct numerical technique for the solution. 
Slater and Wells [3], followed by Shinar and Stcinberg [4], used linear kinematics to model the two-dimensional problem. Accordingly the associated two point boundary value problem (TPBVP) has been solved, and in some cases an analytical solution has been obtained.

Additionally, Shinar, Rosenzstein and Bezner [5], studied a 3-D linearized model by which a solution to the problem was formulated defining the optimal evasive maneuver in three dimensional space.

The optimal control analyses have demonstrated, by applying a more realistic model for the pursuer's dynamics, (such as pure time delay in [3] or some finite order system in [2],[4]) that the evader can guarantee a finite miss-distance even in conflict with a pursuer of unlimited maneuverability, for which a zero miss distance was predicted by the differential game theory [6].

It is important to note that complete information concerning the pursuer's system has been assumed, sometimes implicitly, to be possessed by the evader. This information contains knowledge about the future bchavior of the pursuer (or about its present time derivatives) which is more than generally assumed in the differential game formulation [1]. Consequently, the above result does not necessarily hold for the zero sum differential game even under more realistic models. 


\subsection{Objectives and Motivation}

The optimal control approach for the evasion problem [2-5] indicated the importance of applying a realistic model for the pursuer, and demonstrated that either a finite order system or pure time delay leads to an optimal evasive mancuver that guarantees a non-zero miss-distance.

However, the formulations employed in [2-5] do not treat the evader with the same degree of accuracy as the pursuer. Evader dynamics are either ignored (assuming an ideal system ) or approximated by imposing some bounds.

In the present work our first objective is to provide an analysis for the optimal evasion problem by applying equally realistic models for the pursuer and the evader, and to carry out a parametric study considering the more important parameters of these models and their influence on the optimal strategics and the payoffs.

The second problem to be considered in this work is the optimal evasion with a terminal path angle constraint. The motivation for this problem is derived from cases where the evading vehicle is a missile, guiding toward fixed target, and the pursuer is an interceptor trying to protect this target.Thus, the evasive maneuver is constrained by a terminal path (or heading) angle constraint to guarantee capture of the fixed target. The interceptor is successful if the cvader is destroyed or if it is made to deviate significantly from its course. 
It will be shown that this problem has some interesting features whose importance may exceed the bounds of the pursuit-evasion conflict.

The motivation for the next -and last- problem is self evident. We shall consider the problem of optimal evasion against more than one, starting with two, pursuers. Again the question of guaranteeing a miss-distance shall be investigated allowing the pursuers to select their launch time so as to minimize the closest approach.

The models to be used are all linear, due to the relative complexity of the problems. Consequently, the results will be valid in the vicinity of the nominal collision courses. 


\section{Optimal Evasion Against a Single Pursuer}

\subsection{Basic Assumptions and Equations}

We shall make the following assumptions:

1. The pursuit-evasion conflict is two-dimensional, in the horizontal plane.

2. The speeds of the pursuer (P) and the evader (E) are constant.

3. The trajectories of $P$ and $E$ can be linearized around their collision triangle.

4. P applies a fixed gain proportional navigation .

5. E has complete information on P's system and on the collision course.

6. Each vehicle's acceleration is subject to a first-order lag. 
7. E's lateral acceleration is bounded. (P's lateral acceleration may or may not be bounded.)

It is of importance to note that although the lateral accelerations are state variables, they are by no means small and will not be taken as such in the linearization process.

Referring to Fig 1, by assumptions $1-3$ we get the following equations:

$\sin \left(\gamma_{e_{o}}+\gamma_{e}\right)=\sin \left(\gamma_{e_{o}}\right)+\cos \left(\gamma_{e_{o}}\right) \gamma_{e}$

$\dot{R}=-V_{r}=V_{p} \cos \left(\gamma_{p_{o}}\right)-V_{e} \cos \left(\gamma_{e_{o}}\right)=$ const.,

and

$\dot{y}=\dot{y}_{e}-\dot{y}_{p}=V_{e} \cos \left(\gamma_{e_{o}}\right) \gamma_{e}-V_{p} \cos \left(\gamma_{p_{o}}\right) \gamma_{p}$.

Since the nominal course leads to collision we have the relation :

$V_{e} \sin \left(\gamma_{e_{o}}\right)-V_{p} \sin \left(\gamma_{p_{o}}\right)=0$

P's commanded acceleration is by assumption 4)

$\ddot{y}_{p_{c}}=N^{\prime} V_{r} \dot{\sigma} ; \quad N^{\prime}=N\left(V_{p} / V_{r}\right) \cos \gamma_{p_{o}}$,

where the line of sight (LOS) rate is determined by 
$\dot{\sigma}=\frac{d}{d t}\left(\frac{y}{R}\right)=\frac{y}{V_{r}\left(t_{f}-t\right)^{2}}+\frac{\dot{y}}{V_{r}\left(t_{f}-t\right)}$.

$E$ is free to choose its required acceleration based on the information available to it by assumption 5).

The dynamics of $\mathrm{P}$ is by assumption 6 )

$\frac{d \ddot{y}_{p}}{d t}=\frac{\ddot{y}_{p_{c}}-\ddot{y}_{p}}{\tau_{p}}$

where $\ddot{y}_{p_{c}}$ is $\mathrm{P}^{\prime} \mathrm{s}$ commanded acceleration. A similar equation applics for $\mathrm{E}$. Finally, in applying the last assumption we shall distinguish between $P$ whose actual acceleration shall be bounded by $\ddot{y}_{p_{m}}$ and $E$ whose commanded accelcration shall be bounded (and by the sixth assumption so is its actual acceleration) by $\ddot{y}_{e_{m}}$. Evidently the miss distances under this assumption are smaller than in the other possible combinations of the bounded acceleration problem.

\subsection{Problem Formulation}

We shall define the following state vector $\mathrm{x}$ :

$x=\operatorname{col}\left(\dot{\gamma}_{e}, \gamma_{e}, y, \gamma_{p}, \dot{\gamma}_{p}\right)$ 
and, the control function $u$ :

$u=\frac{\dot{\gamma}_{e_{c}}}{\dot{\gamma}_{e_{m}}}$

where $\ddot{y}_{e_{m}}=\dot{\gamma}_{e_{m}} V_{e} \cos \gamma_{e_{o}}$.

The linear state equations relating $\mathrm{x}$ to $\mathrm{u}$ are :

$\dot{x}_{1}=-\frac{x_{1}}{\tau_{e}}+\dot{\gamma}_{e_{m}} \frac{u}{\tau_{e}}$

$\dot{x}_{2}=x_{1}$

$\dot{x}_{3}=V_{e}^{\prime} x_{2}-V_{p}^{\prime} x_{4}$

$\dot{x}_{4}=x_{5}$

The equation for $x_{5}$ is complicated by the limit imposed on the pursuer's turn rate.

$$
\begin{array}{rlr}
\dot{x}_{5}= & \frac{N^{\prime} V_{e}^{\prime}}{\tau_{p} V_{p}^{\prime}\left(t_{f}-t\right)} x_{2}+\frac{N^{\prime}}{\tau_{p} V_{p}^{\prime}\left(t_{f}-t\right)^{2}} x_{3}- \\
& \frac{N^{\prime}}{\tau_{p}\left(t_{f}-t\right)} x_{4}-\frac{1}{\tau_{p}} x_{5} & \text { for }\left|x_{5}\right|<\dot{\gamma}_{p_{m}} \\
\dot{x}_{5}=0 & \text { for }\left|x_{5}\right|=\dot{\gamma}_{p_{m}}
\end{array}
$$


In the above equations the following notation has been used:

$$
\begin{aligned}
& V_{p}^{\prime}=V_{p} \cos \gamma_{p_{o}} \\
& V_{e}^{\prime}=V_{e} \cos \gamma_{e_{o}}
\end{aligned}
$$

The optimal controi problem is to find $u$ that minimizes the payoff:

$$
J=-x_{3}^{2}\left(t_{f}\right)
$$

subject to the differential equations for $x_{1}(t)-x_{5}(t)$ with specificd initial conditions $x_{1}(0)-x_{5}(0)$ and to the control bound $|u(t)| \leq 1$. The terminal time $t_{f}$ is fixed and is equal to the nominal collision time.

\subsection{Problem Analysis}

We shall define the Hamiltonian :

$H(\lambda, x, u)=\dot{x}_{1} \lambda_{1}+\dot{x}_{2} \lambda_{2}+\dot{x}_{3} \lambda_{3}+\dot{x}_{4} \lambda_{4}+\dot{x}_{5} \lambda_{5}$

where $\dot{x}_{i}$ denotes the right hand side of the associated state equation. The adjoint variables should satisfy:

$$
\dot{\lambda}_{i}=-\frac{\partial H}{\partial x_{i}}
$$


The transversality conditions are :

$\lambda_{i}\left(t_{f}\right)=0$ for $i=1,2,4,5$

$\lambda_{3}\left(t_{f}\right)=-2 x_{3}\left(t_{f}\right)$

And at the discontinuity point $t_{d}$, where the right hand side of $\dot{x}_{5}$ changes, we get

$\lambda_{i}\left(t_{d}+\right)=\lambda_{i}\left(t_{d}-\right) \quad i=1,2,3,4$

$\lambda_{5}\left(t_{d}+\right)=\lambda_{5}\left(t_{d}-\right)+c$

$H\left(t_{d}+\right)=H\left(t_{d}-\right)$.

A derivation of the maximum principle for control problems with discontinuous system of equations is given in [10](sce pp.311-312). The optimality condition requires

$u(t)=-\operatorname{sgn} \lambda_{1}(t)$

Hence, $\lambda_{1}$ is the control switching function. (The linearity of the system rules out singular arcs.) Thus, the solution is a bang-bang type of control for E's commanded accelcration similarly to the ideal system case [4], where its actual acceleration was bang-bang. 


\subsection{Computational Results}

The solutions for the TPBVP were obtained numerically using a multiple shooting algorithm (MSA)[11]. The initial states are all zero and the following numerical values are used:

$$
\begin{aligned}
& N^{\prime}=3 \\
& V_{p}^{\prime}=1000 \text { f.p.s. } \\
& V_{e}^{\prime}=500 \text { f.p.s. } \\
& \dot{\gamma}_{e_{m}}=0.3 \mathrm{rad} / \mathrm{sec} \\
& \tau_{p}=0.5 \mathrm{sec} \\
& t_{f}=3.75 \mathrm{sec}
\end{aligned}
$$

A two-parameter family of solutions has been obtained by varying $\left(\ddot{y}_{p_{m}} / \ddot{y}_{e_{m}}\right)$ and $\left(\tau_{e} / \tau_{p}\right)$ i.e. the relative maneuverability and the relative time response of the opponents are used as parameters. The miss-distance results are presented as a function of these parameters in Fig 2. Note that E's time constant need not be smaller than P's for evading with a significant miss distance, and that a non-zero miss distance is guaranteed even when the pursuer has unlimited maneuverability. 
The problem as formulated has a closed form solution for $\tau_{e}=0, \ddot{y}_{p_{m}}=\infty$ which has been obtained in [4]. For this case E applies a bang-bang type of control with two switching points, (for $N^{\prime}=3$ ), the first of which is at time $t=0$ and the second is at $0 \equiv \frac{t_{f}-t}{\tau_{p}}=2$. For other values of $\tau_{e}$ and $\ddot{y}_{p_{m}}$ the solution still has two switching points, the first is at $t=0$ and the corresponding $\theta$ parameter is shown in Fig 3. In all these results the guidance parameter $N^{\prime}$ is three. In [4] E's system was approximated by a ramp function with $t_{r}$ as the ramp time ( the minimum time to change the lateral acceleration from $-\ddot{y}_{e_{m}}$ to $\ddot{y}_{e_{m}}$ ). Their results may be compared to ours by approximating :

$t_{r}=3 \tau_{e}$

In general the comparison is satisfactory for both the miss distance and the switching point.For "slow" evader $\left(\tau_{e} / \tau_{p}>1\right)$ but with relativcly high maneuverability $\left(\ddot{y}_{e_{m}} / \ddot{y}_{p_{m}}>0.25\right)$ the ramp time approximation results are more optimistic (for $E$ ) and the predicted miss distances are greater than the first order model ones. For "fast" evader and/or for less maneuverable one the results are in a very good agreement. 


\section{Optimal Evasion With a Path Angle Constraint}

\subsection{Problem Formulation}

We shall continue to employ assumptions 1)-5) of the previous section. However, assumptions 6) and 7) will be further restricted to:

$\left.6^{\prime}\right) \mathrm{P}$ is a first order system, $\mathrm{E}$ is an ideal system (no time lag)

7') E's lateral acceleration is bounded while P's is unbounded.

As will be shown, these assumptions simplify the problem by reducing the dimension of the model.

Fig 4 presents the relative geometry of the problem. T is a fixed target to which $\mathrm{E}$ is homing. $\mathrm{P}$ is an interceptor that defends $\mathrm{T}$. Therefore $\mathrm{E}^{\prime} \mathrm{s}$ mission in gencral is to first evade from $\mathrm{P}$ and then to destroy T. If, however, EC is larger than ET ( $\mathrm{C}$ is the collision point ) then the evasion problem is irrelevant. 
In general the heading error of E's velocity vector from its line of sight to $T$ will determine the miss-distance of its homing mission. Thus, our linear model implies a terminal path (or heading )angle constraint on the optimal evasion problem.

The state vector for this case is :

$x=\operatorname{col}\left(\gamma_{e}, y, \gamma_{p}, \dot{\gamma}_{p}\right)$

and, the control $\mathrm{u}$ :

$u=\frac{\dot{\gamma}_{e}}{\dot{\gamma}_{e_{m}}}$

The linear state equations relating $\mathrm{x}$ to $\mathrm{u}$ are :

$\dot{x}_{1}=\dot{\gamma}_{e_{m}} u$

$\dot{x}_{2}=V_{e}^{\prime} x_{1}-V_{p}^{\prime} x_{3}$

$\dot{x}_{3}=x_{4}$ 


$$
\begin{gathered}
\dot{x}_{4}=\frac{N^{\prime} V_{e}^{\prime}}{\tau_{p} V_{p}^{\prime}\left(t_{f}-t\right)} x_{1}+\frac{N^{\prime}}{\tau_{p} V_{p}^{\prime}\left(t_{f}-t\right)^{2}} x_{2}- \\
\frac{N^{\prime}}{\tau_{p}\left(t_{f}-t\right)} x_{3}-\frac{1}{\tau_{p}} x_{4}
\end{gathered}
$$

The optimal problem therefore is to find $u$ that minimizes the payoff $J=-x_{2}^{2}\left(t_{f}\right)$ subject to $|\mathrm{u}(\mathrm{t})| \leq 1$ and to $\left|\gamma_{e}\left(t_{f}\right)\right| \leq \gamma_{f}$. The terminal time is fixed and is determined by the nominal collision time of $\mathrm{P}$ and $\mathrm{E}$.

\subsection{Problem Analysis}

We shall define the Hamiltonian:

$H(\lambda, x, u)=\dot{x}_{1} \lambda_{1}+\dot{x}_{2} \lambda_{2}+\dot{x}_{3} \lambda_{3}+\dot{x}_{4} \lambda_{4}$

The adjoint variables should satisfy the following equations :

$\dot{\lambda}_{i}=-\frac{\partial H}{\partial x_{i}}$

The transversality conditions for this problem are [9]:

$\lambda_{i}\left(t_{f}\right)=0$ for $i=3,4$

$\lambda_{2}\left(t_{f}\right)=-2 x_{2}\left(t_{f}\right)$ 


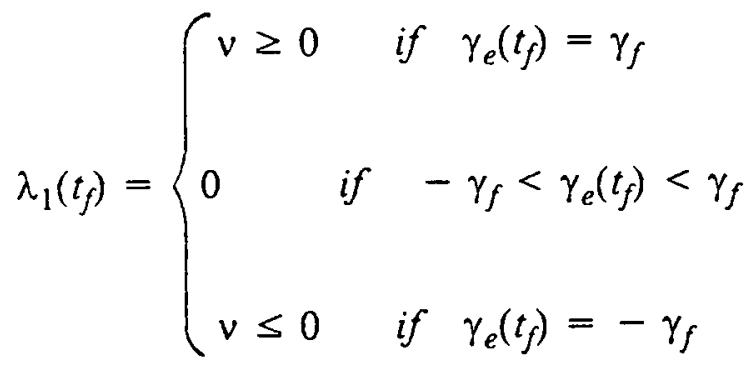

The last condition is a Kuhn-Tucker like condition for our inequality endconstraint which implics that violating the constraint would yicld a better performance indcx.

From the maximum principle we get (similarly to the previous section)

$u(t)=-\operatorname{sgn} \lambda_{1}(t)$

Hence, $\lambda_{1}$ is the control switching function. Thus, the solution is a bang-bang type of control for E's actual acceleration.

The problem without the path-angle constraint was solved analytically in [4] by Laplace transforms. For this problem $\lambda_{1}\left(t_{f}\right)=0$

Let $\theta=\left(t_{f}-t\right) / \tau_{p}$. (normalized time to go). The adjoint equations are :

$\frac{d \lambda_{1}}{d 0}=\left(\tau_{p} V_{e}^{\prime}\right) \lambda_{2}+\left(\frac{N^{\prime} V_{e}^{\prime}}{\tau_{p} V_{p}^{\prime}{ }_{p}^{0}}\right) \lambda_{4}$

$\frac{d \lambda_{2}}{d \theta}=\left(\frac{N^{\prime}}{\tau_{p}^{2} V_{p}^{\prime} \theta^{2}}\right) \lambda_{4}$ 
$\frac{d \lambda_{3}}{d 0}=-\left(\tau_{p} V_{p}^{\prime}\right) \lambda_{2}-\left(\frac{N^{\prime}}{\tau_{p} 0}\right) \lambda_{4}$

$\frac{d \lambda_{4}}{d \theta}=\tau_{p} \lambda_{3}-\lambda_{4}$

The solution for $\lambda_{1}(\theta)$ has the form

$\bar{\lambda}_{1}(\theta)=p(\theta) \exp (-\theta)$,

where $p$ is a polynomial of order $N^{\prime}-1$ (for an integer effective gain $N^{\prime}$ ). Consequently there are $\mathrm{N}^{\prime}-1$ switching points (including the final time). The more general result (for any $\mathrm{N}^{\prime}$, integer or not) is the inverse Laplace transform of :

$\vec{\Lambda}_{1}(s)=\frac{s^{N-2}}{(s+1)^{N}}$

This result is valid for our problem only if

$\left|\gamma_{e}\left(t_{f}\right)\right|=\dot{\gamma}_{e_{m}}\left|\int_{0}^{t_{0}} \operatorname{sgn} \bar{\lambda}_{1}(t) d t\right|<\gamma_{f}$

We now consider the case where the terminal path angle constraint is binding and note that the solution for the last three adjoint equations is unchanged ( since this set is independent of the the first co-state variable). However, the first co-state (the switching function) is now

$\lambda_{1}(\theta)=\bar{\lambda}_{1}(\theta)+v$, 
where $v$ will be determined by

$$
-\int_{0}^{t} \operatorname{sgn}\left(\bar{\lambda}_{1}+v\right) d t=\frac{\gamma_{f}}{\dot{\gamma}_{e_{m}}} \text {. }
$$

Consequently, the solution contains up to $\mathrm{N}^{\prime}$ switching points that are the zeros of $\lambda_{1}$. ( $\mathrm{N}^{\prime}-1$ from the polynomial term and an additional one from the exponential term.) Since these zeros determine its integrand, the last integral is continuous with $v$ (from the continuous behavior of the roots) but is not, in general, differentiable. Consequently the implicit function theorem is not applicable and the solution for $v$ may not be unique.

In Fig 5 two alternatives for the solution are presented $\left(\mathrm{N}^{\prime}=3\right)$. Both of them satisfy the terminal constraint and both are locally optimal, however only one of these is the global optimum. The other solution can be climinated by the terminal sign of $\lambda_{1}$ (i.e. the sign of $u(t)$, as required by the transversality conditions). 


\subsection{Computational Results and Problem Decomposition}

The problem was solved using the data given in 2.3 for two terminal times : $t_{f}=10 \tau_{p}$ and $t_{f}=6 \tau_{p}$. Fig 6 and Fig 7 present the payoff as a function of the terminal constraint, while Fig 8 and Fig 9 present the switching points of the optimal control function for those values.

As it is shown in Fig 7 for $t_{f}>>\tau_{p}$ the original switching point hardly moves as the constraint is tightened and the additional switching point is the one that is responsible for satisfying the path angle constraint. This fact is true in general for any $N^{\prime}$.

To explain this interesting phenomena we shall present two considerations: the first will be purely mathematical and the second will be of a more physical and heuristic type.

The adjoint system analysis, as indicated in [4], interprets $\bar{\lambda}_{1}$ as the influence function of the control $u$ on the miss distance. From the Bode representation of $\bar{\lambda}_{1}$ (Fig 10) we conclude that it behaves as a band-pass filter, hence the low frequency content of the control has little effect on the payoff. The path-angle is an integral of the control and is effected by the control history from $t_{0}$ onward(as a low-pass filter). Thus, in the optimal control the constraint is satisficd by appropriatetly selecting the early switching point. Altering the constraint causes the early switching point to move but has little effect on the late switches. 
The physical interpretation is more obvious. The basic principle of the evasion maneuver, under our assumptions, is to take advantage of the finite time lag of $P$, hence only the last few time-constants are of importance. E however, knows "the future" (assumption 5 ) so it can adjust the terminal path angle to the constraint by adding the initial switching point without significantly affecting the payoff.

As a result, the constrained solution can be composed from the optimal unconstrained one, plus one more switching point to satisfy the constraint. Unless $t_{f}$ is of the same order as $\tau_{p}$ this will be a good approximation for the solution. Moreover, we can conclude from Fig 10 that the higher is $\mathrm{N}^{\prime}$ the better will be the approximated solution. 


\section{Optimal Evasion Against Two (or more)}

\section{Pursuers.}

\subsection{Problem Formulation}

We shall consider the problem of optimal evasion against two guided missiles under the simplifying assumptions of the previous chapter for each of the pursuers and for the evader. Thus, each pursuer constructs its own collision triangle around which we may linearize the kinematics.

For simplicity we will further assume that the pursuers are identical i.e. they have the same time delay $\tau_{p}$ and the same gain $N^{\prime}$.

Let $\Delta$ denotes the nominal time difference between impacts, let $P_{2}$ be the first of the two pursucrs and let the state vector be

$x=\operatorname{col}\left(\gamma_{e}, y_{1}, \gamma_{p_{1}}, \dot{\gamma}_{p_{1}}, y_{2}, \gamma_{p_{2}}, \dot{\gamma}_{p_{2}}\right)$ 
and the control $\mathrm{u}$

$$
u=\frac{\dot{\gamma}_{e}}{\dot{\gamma}_{e_{m}}}
$$

The linear state equations relating $\mathrm{x}$ to $\mathrm{u}$ are :

$$
\begin{aligned}
& \dot{x}_{1}=\dot{\gamma}_{e_{m}} u \\
& \dot{x}_{2}=V_{e}^{\prime} x_{1}-V_{p_{1}}^{\prime} x_{3} \\
& \dot{x}_{3}=x_{4} \\
& \dot{x}_{4}=\frac{N^{\prime} V_{e}^{\prime}}{\tau_{p} V_{p_{1}}^{\prime}\left(t_{f}-t\right)} x_{1}+\frac{N^{\prime}}{\tau_{p} V_{p_{1}}^{\prime}\left(t_{f}-t\right)^{2}} x_{2}- \\
& \frac{N^{\prime}}{\tau_{p}\left(t_{f}-t\right)} x_{3}-\frac{1}{\tau_{p}} x_{4} \\
& \dot{x}_{5}=\left(V_{e}^{\prime} x_{1}-V_{p 2}^{\prime} x_{6}\right) h\left(t_{f}-\Delta-t\right) \\
& \dot{x}_{6}=\left(x_{7}\right) h\left(t_{f}-\Delta-t\right)
\end{aligned}
$$




$$
\begin{aligned}
\dot{x}_{7}=\frac{N^{\prime} V_{e}^{\prime}}{\tau_{p} V_{p_{2}}^{\prime}\left(t_{f}-t-\Delta\right)} h\left(t_{f}-\Delta-t\right) x_{1} \\
+\frac{N^{\prime}}{\tau_{p} V_{p_{2}}^{\prime}\left(t_{f}-t-\Delta\right)^{2}} h\left(t_{f}-\Delta-t\right) x_{5}- \\
\quad \frac{N^{\prime}}{\tau_{p}\left(t_{f}-t-\Delta\right)} h\left(t_{f}-\Delta-t\right) x_{6}-\frac{1}{\tau_{p}} h\left(t_{f}-\Delta-t\right) x_{7}
\end{aligned}
$$

Where $h(z)$ is defined by the Heaviside step function

$$
h(z)= \begin{cases}1 & \text { if } z \geq 0 \\ 0 & \text { if } z<0\end{cases}
$$

Hence, after $P_{2}$ finishes its mission, only the first 4 equations remain active .

The optimal control problem is to find $u$ that minimizes $J$,

$J=-y_{1}^{2}\left(t_{f}\right)$

subject to

$$
|u| \leq 1
$$


and to

$y_{2}^{2}\left(t_{f}-\Delta\right)=c$

where $0 \leq c \leq y_{m}^{2}$, and $y_{m}^{2}$ is the optimal performance index in the case of the single pursuer.Consequently, a simple survey over the parameter $\mathrm{c}$ will provide the solution for the problem of maximizing the smaller of the two miss distances in our case.

The time difference $\Delta$ will also be a parameter which may be adjusted by the pursuers to maximize J. (We may think of two A/A missiles launched by the same aircraft.)

\subsection{Problem Analysis}

We shall define the Hamiltonian to be

$H(\lambda, x, u)=\dot{x}_{1} \lambda_{1}+\dot{x}_{2} \lambda_{2}+\dot{x}_{3} \lambda_{3}+\dot{x}_{4} \lambda_{4}+\dot{x}_{5} \lambda_{5}+\dot{x}_{6} \lambda_{6}+\dot{x}_{7} \lambda_{7}$

and introduce, as before, the non-dimensional time to go

$\theta=\frac{\left(t_{f}-t\right)}{\tau_{p}}$

The adjoint system is now (redefining $\Delta$ to be in $\tau_{p}$ units) 


$$
\begin{aligned}
& \frac{d \lambda_{1}}{d \theta}=\left(\tau_{p} V_{e}^{\prime}\right) \lambda_{2}+\left(\frac{N^{\prime} V_{e}^{\prime}}{\tau_{p} V_{p_{1}}^{\prime} \theta}\right) \lambda_{4}+\tau_{p} V_{e}^{\prime} h(\theta-\Delta) \lambda_{5}+\frac{N^{\prime} V_{e}^{\prime} h(\theta-\Delta)}{\tau_{p} V_{p_{2}}^{\prime}(0-\Delta)} \lambda_{7} \\
& \frac{d \lambda_{2}}{d \theta}=\left(\frac{N^{\prime}}{\tau_{p}^{2} V_{p_{1}}^{\prime} \theta^{2}}\right) \lambda_{4} \\
& \frac{d \lambda_{3}}{d 0}=-\left(\tau_{p} V_{p_{1}}^{\prime}\right) \lambda_{2}-\left(\frac{N^{\prime}}{\tau_{p} \theta}\right) \lambda_{4} \\
& \frac{d \lambda_{4}}{d \theta}=\tau_{p} \lambda_{3}-\lambda_{4} \\
& \frac{d \lambda_{5}}{d \theta}=\frac{N^{\prime} h(\theta-\Delta)}{\tau_{p}^{2} V_{p_{2}}^{\prime}(\theta-\Delta)^{2}} \lambda_{7} \\
& \frac{d \lambda_{6}}{d \theta}=-\tau_{p} V_{p_{2}}^{\prime} h(\theta-\Delta) \lambda_{5}-\frac{N^{\prime} h(\theta-\Delta)}{\tau_{p}(\theta-\Delta)} \lambda_{7} \\
& \frac{d \lambda_{7}}{d \theta}=\tau_{p} h(0-\Delta) \lambda_{6}-h(\theta-\Delta) \lambda_{7}
\end{aligned}
$$

The transversality conditions for our case are :$$
\lambda_{i}(0)=0 \text { for } i \neq 2
$$$$
\lambda_{2}(0)=-2 y_{1}(0)
$$ 
At the time of the first nominal impact $\theta=\Delta$ we shall have the following conditions:

$\lambda_{i}(\Delta+)=\lambda_{i}(\Delta-) \quad$ for $i \neq 5$

$\lambda_{5}(\Delta+)=\lambda_{5}(\Delta-)+v$

From the maximum principle, as in the preceding sections, the first adjoint parameter $\lambda_{1}$ is the switching function. Notice that the adjoint equations 2-4 and 5-7 are independent, thus the solution described in 3.2 applies to both. Moreover the solution for the first adjoint variable is a superposition of the form:

$\lambda_{1}=c_{1} p(\theta) \exp (-\theta)+c_{2} p(\theta-\Delta) \exp (-\theta+\Delta) h(\theta-\Delta)$

To illustrate the solution we let $\mathrm{N}^{\prime}=3$ and $V_{p_{1}}^{\prime}=V_{p_{2}}^{\prime}=V_{p .}$, then the solution for the co-state vector is:

$\lambda_{1}=c_{1} \tau_{p} V_{p} \exp (-\theta)\left(\theta-\theta^{2} / 2\right)+c_{2} \tau_{p} V_{p} \exp (\Delta-\theta)\left((0-\Delta)-(\theta-\Delta)^{2} / 2\right) h(0-\Delta)$

$\lambda_{2}=c_{1} \exp (-\theta)(1-\theta / 2)$

$\lambda_{3}=-c_{1} V_{p} \tau_{p} \exp (-\theta)\left(\theta-\theta^{2} / 2\right)$

$\lambda_{4}=-c_{1} \tau_{p}^{2} V_{p} \exp (-0)\left(\theta^{2} / 2-\theta^{3} / 6\right)$

$\lambda_{5}=c_{2} \exp (\Delta-0)(1-(\theta-\Delta) / 2) h(0-\Delta)$

$\lambda_{6}=-c_{2} V_{p} \tau_{p} \exp (\Delta-0)\left((\theta-\Delta)-(\theta-\Delta)^{2} / 2\right) h(\theta-\Delta)$ 
$\lambda_{7}=-c_{2} V_{p} \tau_{p}^{2} \exp (\Delta-\theta)\left(\exp (\Delta-\theta)^{2} / 2+\exp (\Delta-\theta)^{3} / 6\right) h(0-\Delta)$

We may draw now two conclusions regarding the switching points. First, there will be no more than $2\left(\mathrm{~N}^{\prime}-1\right)$ of them, and the second conclusion is that any switching point of the single pursuer case that takes place after $t_{f}-\Delta \tau_{p}$ will be also a switching point for our present case. Both the conclusions may bc verified by checking the roots of $\lambda_{1}$.

We can use now a simple parameter optimization procedure to find out the exact location of the switching points, from which the miss-distance may be obtained, as suggested in [4], by the simple relation

$y_{1}\left(t_{f}\right)=\tau_{p}^{2} \ddot{y}_{e_{m}}\left(g\left(0_{0}\right)+2 \sum_{i} g\left(\theta_{i}\right)\right)$,

where

$g(x)=\int_{0}^{x} \bar{\lambda}_{1}\left(x^{\prime}\right) d x^{\prime}$

This relation is based on the observation made about $\bar{\lambda}_{1}$ ( the solution for the single pursuer case ) as the impulse response function relating control to missdistance. In general, as indicated in [4], the first term in the above expression for the miss distance can be neglected because of strong damping of $\exp \left(-0_{0}\right)$. 


\subsection{Computational Results}

The solution can be completed by employing a parameter optimization technique to locate the switching points. To illustrate the procedure let $\mathrm{N}^{\prime}=3$, thus the payoff to be maximized is

$J=\left(\sum_{i 1}^{3}(-1)^{i} \exp \left(-0_{i}\right) \theta_{i}^{2}\right)^{2}$

where $0_{i}$ is the $\mathrm{i}$-th switching point and $\mathrm{J}$ is the the square of the miss distance of $P_{1}$. However, this should be subjected to a constaint on the other miss distance

$\left(\sum_{i 1}^{3}(-1)^{i} \exp \left(\Delta-\theta_{i}\right)\left(\Delta-\theta_{i}\right)^{2} h\left(\theta_{i}-\Delta\right)\right)^{2}-c \geq 0$.

Notice that both the payoff and the constraint are analytically differentiable . Fig 11 presents the results (payoff versus constraint) for various $\Delta$ as obtained by an optimization program which employs a gradient projection algorithm [12]. The optimal switching points as obtained by the program are in agrecment with the prediction of the previous section.

As expected, the payoff is changed monotonically with the constraint. In order to maximize the closest approach of the pursucrs we need to take the results from the 45 degree line on which the two miss - distances are the same. 
Fig 12 presents the optimal miss distance against $\Delta$. Notice the existence of an optimum $\Delta$, from the pursuer point of view, for which the maximal miss distance is minimized. The results for $\mathrm{N}^{\prime}=4$ have been obtained in the same way and a different optimal $\Delta$ was found as illustrated in Fig 12. For more than two pursuers the analysis is virtually the same, and we end up with $n\left(N^{\prime}-1\right)$ as the maximal number of switching points in the case of $n$ pursuers. 


\section{Conclusions}

By applying linearized kinematics to the optimal evasion problem solutions for some relatively complicated cases have been obtained. The optimal commanded lateral acceleration is, in general, a 'bang- bang' non-singular one governed by a switching function. The number of switching points and thcir location is dependent on the dynamics of the pursuer and the evader and on the particular constraints of the problem in hand.

The validity of the results is restricted by the assumption set given in section 2.1. It is of interest to examine briefly the sensitivity of the solution to those assumptions.

The first assumption restricted the discussion to the planar case. In [5] the 3-D case was investigated and as it turns out the optimal strategy may be decomposed into two phases. The first is lift orientation to an optimal plane, and the second 
phase comprises of 'bang-bang' maneuvers of 180 deg bank angle (for a bank to turn vehicle) governed by a switching function similar to the 2-D one.

The second assumption fixed the speed of the opponents. Removing this assumption may change the nature of the solution since in general the control function does not appear linearly in the state equations. However, the solution may still be valid for the last few time-constants where the velocity change may be negligible. This is an important problem which requires further investigation, since in many realistic situations the constant speed assumption is not applicable.

In removing the third assumption, which allowed us to linearize the state equations, we get either a 'bang-bang' type of solution [2],[4],[8] or a singular behavior.In general, the computations are significantly more involved.

Assumption (4) imposed the fixed-gain proportional navigation strategy for the persuer. In general, different pursuit strategies imply different evasion strategies and therefore the results for the proportionally guided pursuer problem are not applicable to other problems.

In assumption (5) we required that $E$ has complete information on P. Fig 13 presents the sensitivity of the expected payoff to the uncertainty in the terminal time. A uniform distribution between $t_{f}-\Delta$ and $t_{f}+\Delta$ has been assumed and the optimal deterministic strategy was applied (assumptions 6 and 7 were replaced by $6^{\prime}$ and $7^{\prime}$ for simplicity) using the mean value. The results of this sim- 
ple analysis are sufficient to illustrate the importance of this assumption. Full stochastic treatment should be employed once we decide to remove it.

In the sixth assumption a first order dynamic model has been assumed for $\mathrm{P}$ and E. In [4] it was found out that changing the order of P's system has a slight affect on the switching points but the payoff is significantly increased as the order of P's system increases. No analogous analysis has been done yet for E's system.

It is of interest to release the fixed control bounds imposed by the seventh assumption and to introduce velocity dependent bounds (for non-constant speed cases). This has been done for the differential game approach [7], and its application to the one sided optimal control formulation is recommended for future investigation of this approach. 


\section{References}

1. Isaacs, R., Differential Games, John Wiley and Sons, New York,1965.

2. Julich,P.M. and Borg,D.A,"Proportional Navigation vs an Optimally Evading Constant- Speed Target in Two Dimensions,"Journal of Spacceraft and Rockets ,Dec. 70 , pp.1454-1457.

3. Slater,G.L. and Wells,W.R.,"Optimal Evasive Tactics with Time Delay,"Journal of Spacecraft and Rockets, Vol 10,May 1973, pp.309-313.

4. Shinar,J. and Steinberg,D.,"Analysis of Optimal Evasive Maneuvers Based on a Linearized Two-Dimensional Model," Journal of Aircraft ,Vol 14,Aug.1977,pp.795-802.

5. Shinar ,J. ,Rosenzstein,Y. and Bezner,E.,"Analysis of Three Dimensional Optimal Evasion with Linearized Kincmatics,"Journal of Guidance and Control ,Vol 2,Sept.1979, ,pp. 353-360.

6. Gutman,S. and Leitmann,G.,"Optimal Strategies in the Neighborhood of a Collision Course," AIAA Journal, Vol 14, Sept.1976, pp.1210-1212.

7. Jarmark,B.S.A., Mcrz,A.W. and Breakwell,J.V. , "The Variable - Speed TailChase Aerial Combat Problem ," J. Guidance and Control, May-June 1981, pp 323-328.

8. Forte ,I., Steinberg A., and Shinar, J.,"The Effect of Non-Linear Kinematics in Optimal Evasion," Optimal Control Applications \& Methods, Vol 4, 1983,pp 139-152. 
9. Bryson,A.E. and Ho ,Y. C. , Applied Optimal Control, Hemisphere Publishing Co., Washington ,1969.

10. Pontryagin,L.S. et al. , The Mathematical Theory of Optimal Processes, Interscience, New York, 1962.

11. Bulirsch, R.,"Einfuhrung in die Flungbahnoptimatierung die Mehrzielmethode zur Numerischen Losung von Nichtlinearen Randwertproblemen und Aufgaben der Optimalenn Stcuerung ," Lehrgang Flugbahnoptimierung Carl-Cranz-Gesellschaft e.v. ,October 1971.

12. Kelley,H.J.,and Speyer, J.L.,"Accelerated Gradient Projection," Lecture Notes on Mathematics 132 ,Springer-Verlag, Berlin, 1970. 


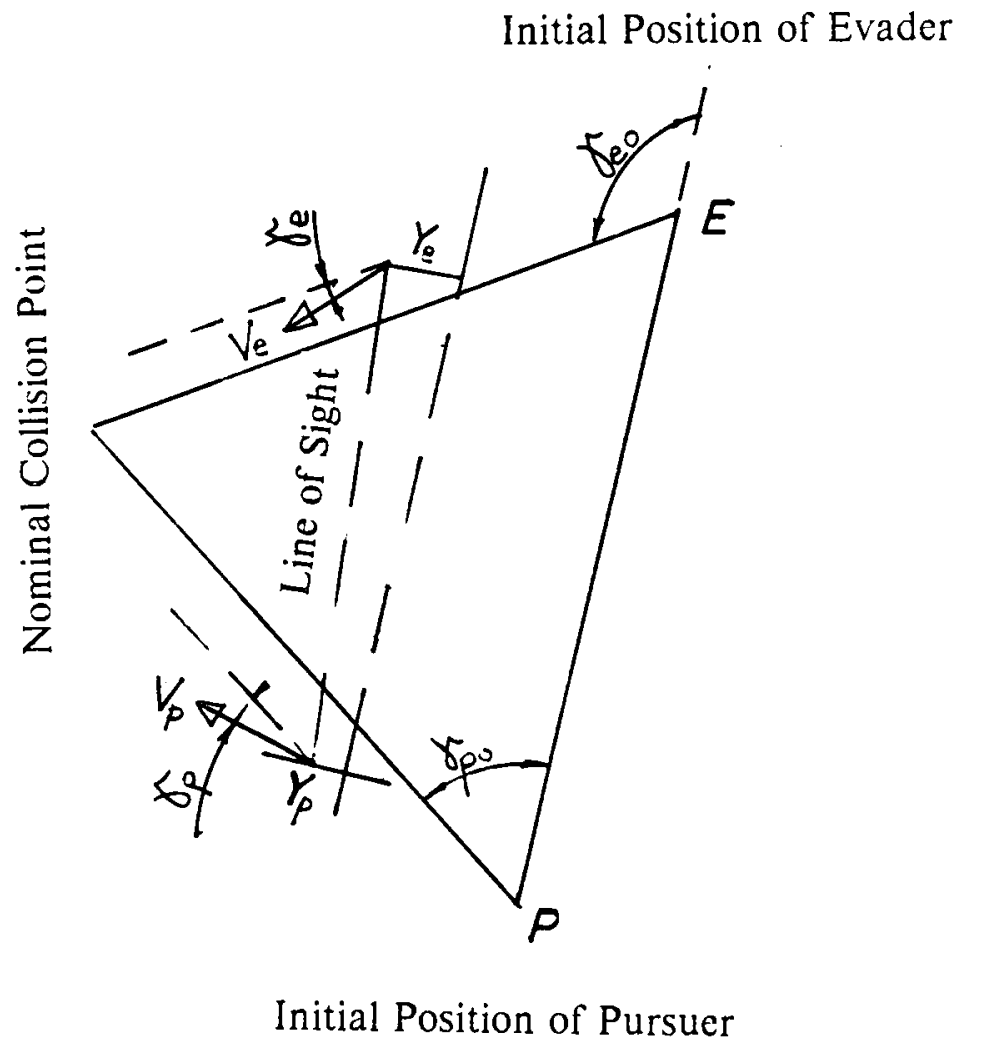

Figure 1: Problem Geometry 


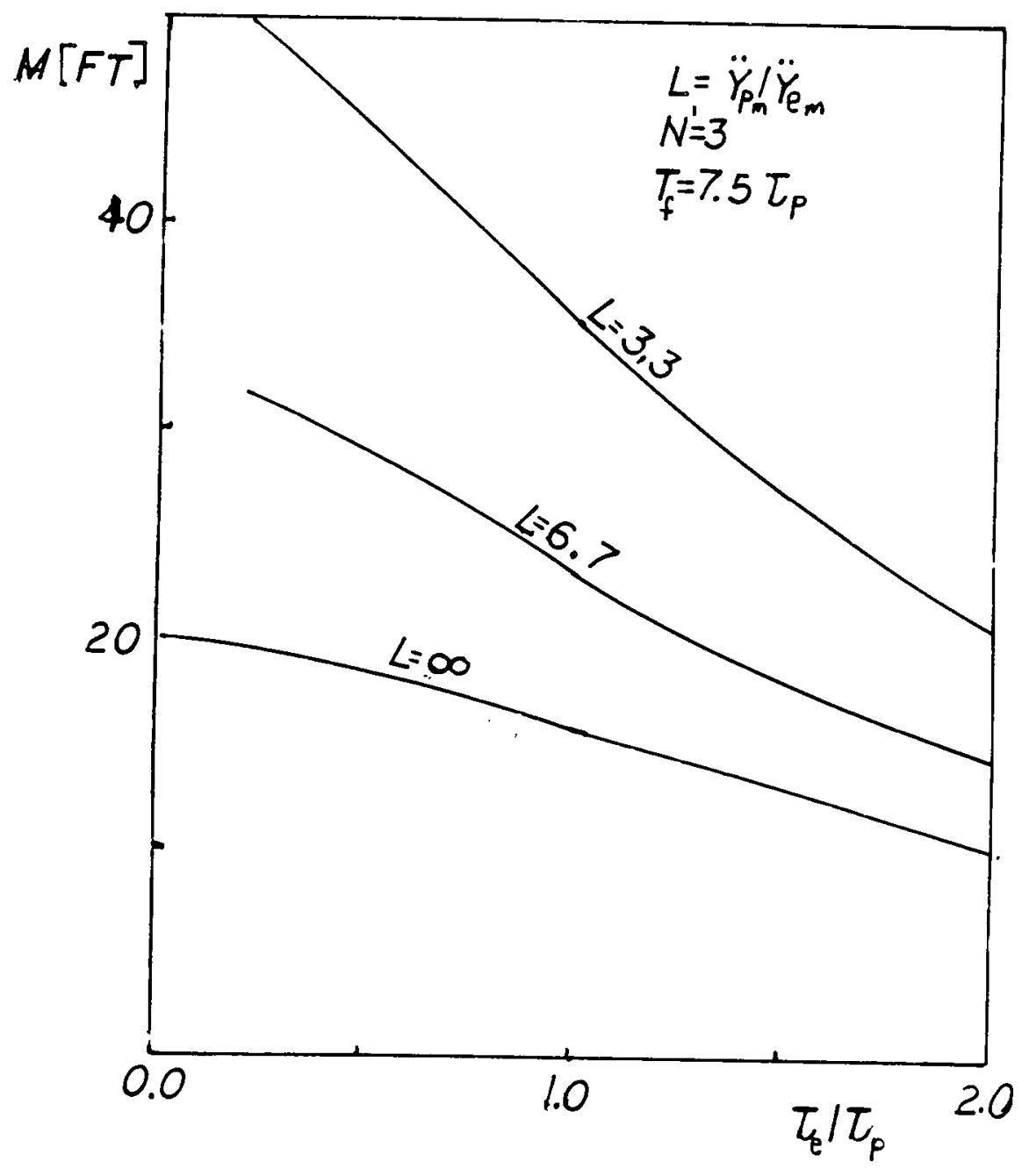

Figure 2: Miss Distance versus Time Lag Ratio 


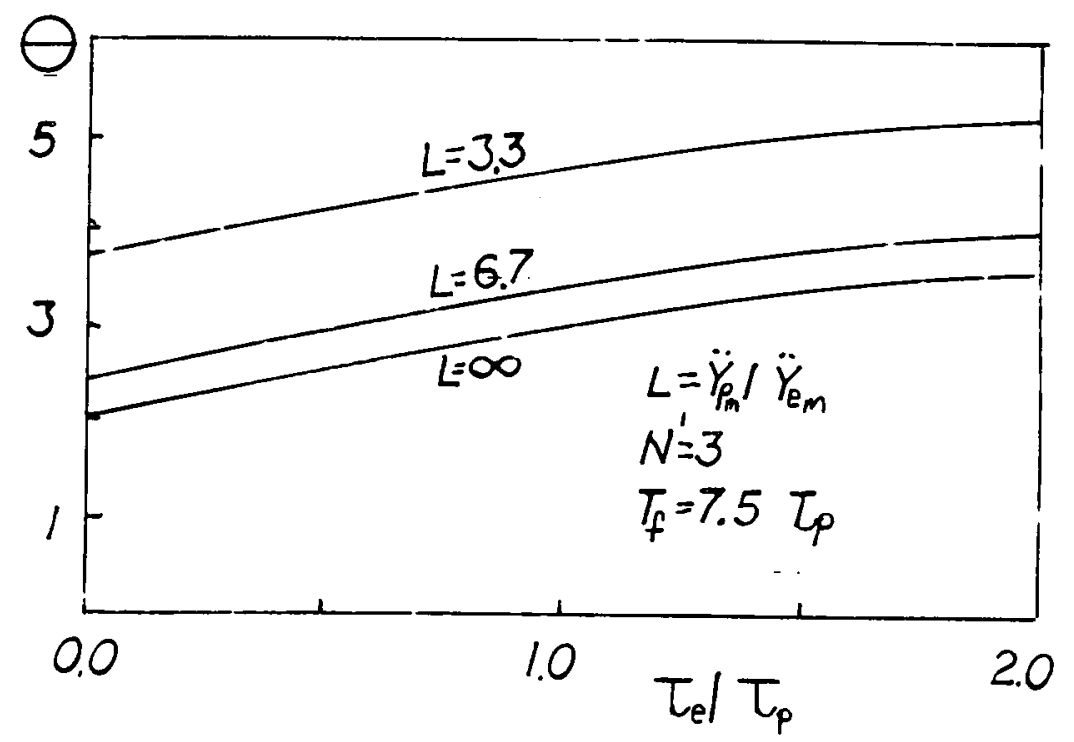

Figure 3: Switching Point versus Time Lag Ratio 


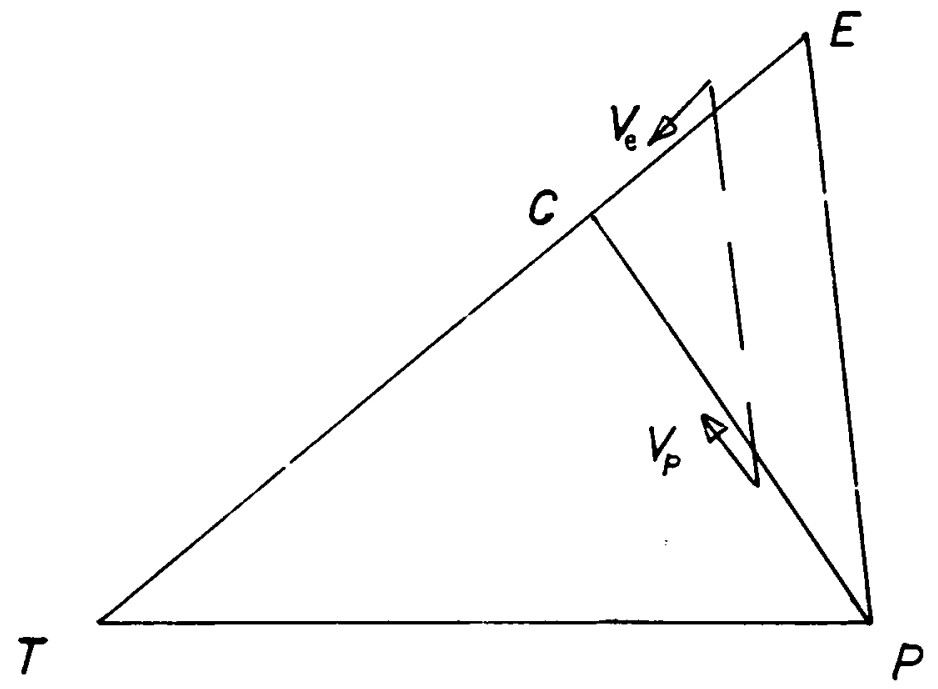

Figure 4: Constrained Problem Geometry 

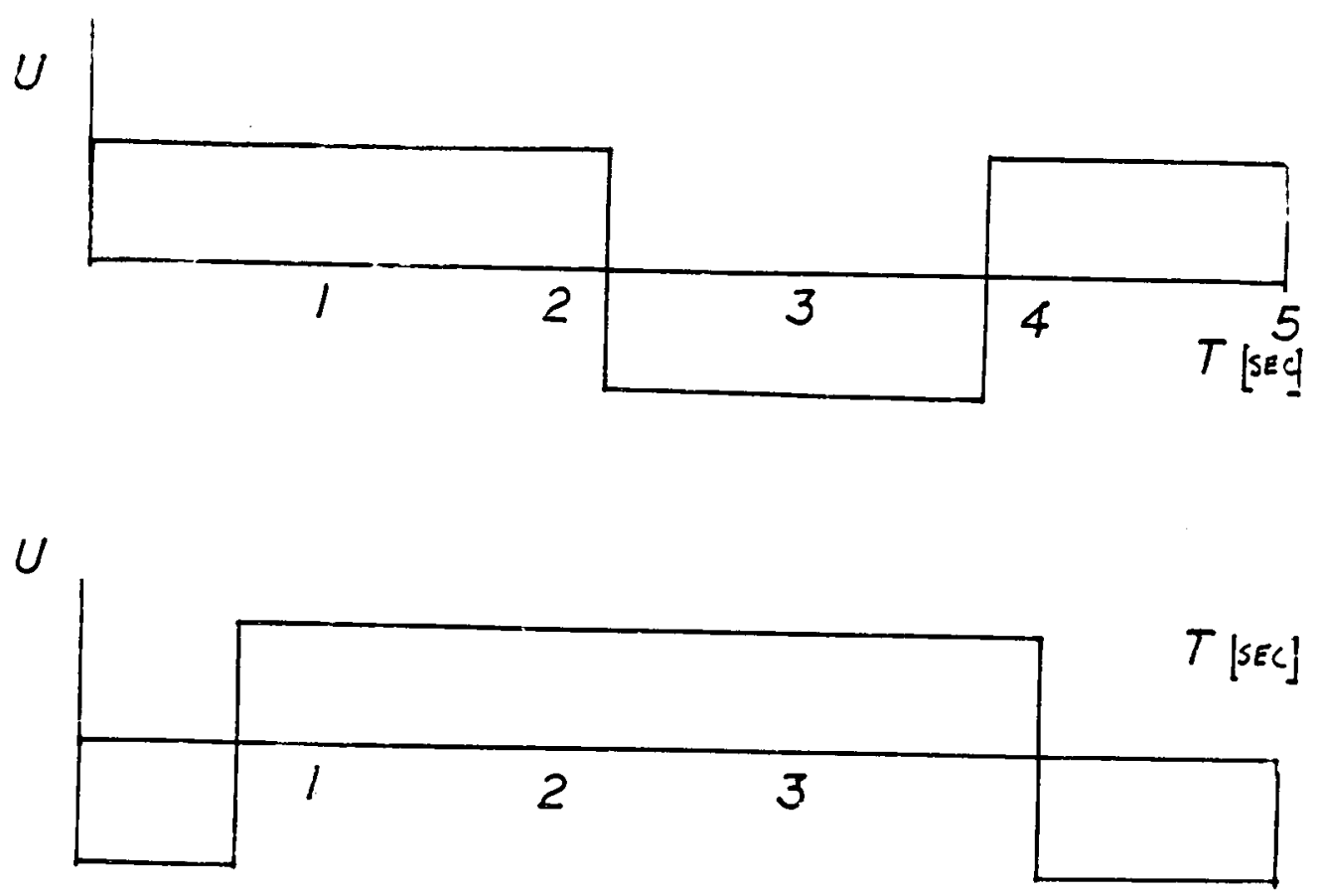

Figure 5: Control Options for the Constrained Problem 


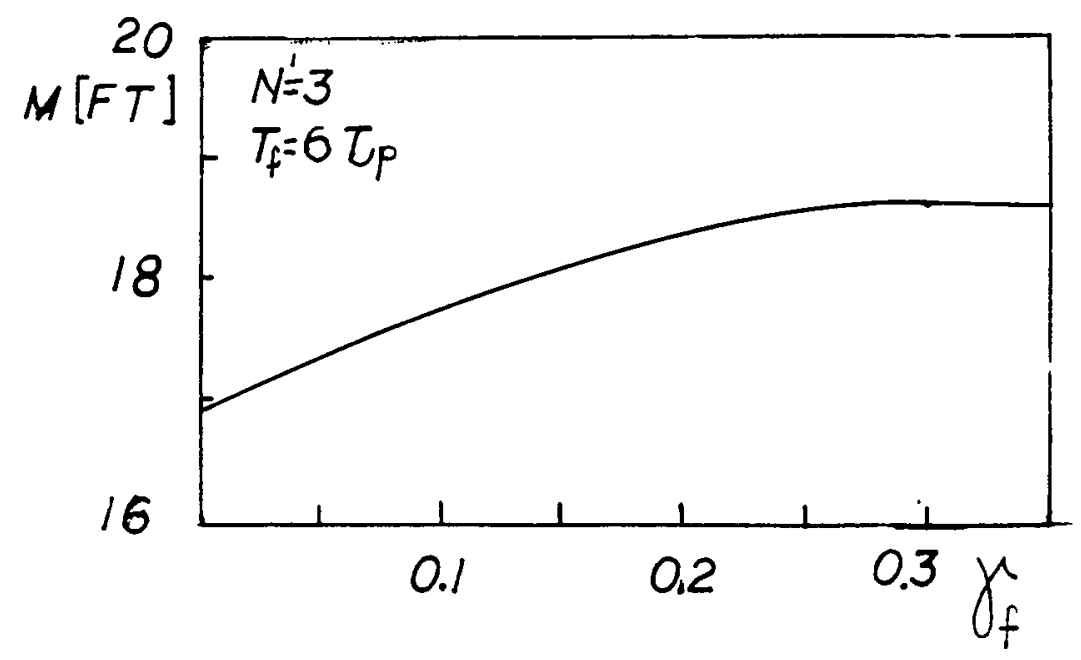

Figure 6: Miss Distance versus Terminal Path Angle for $t_{f}=6 \tau_{p}$ 


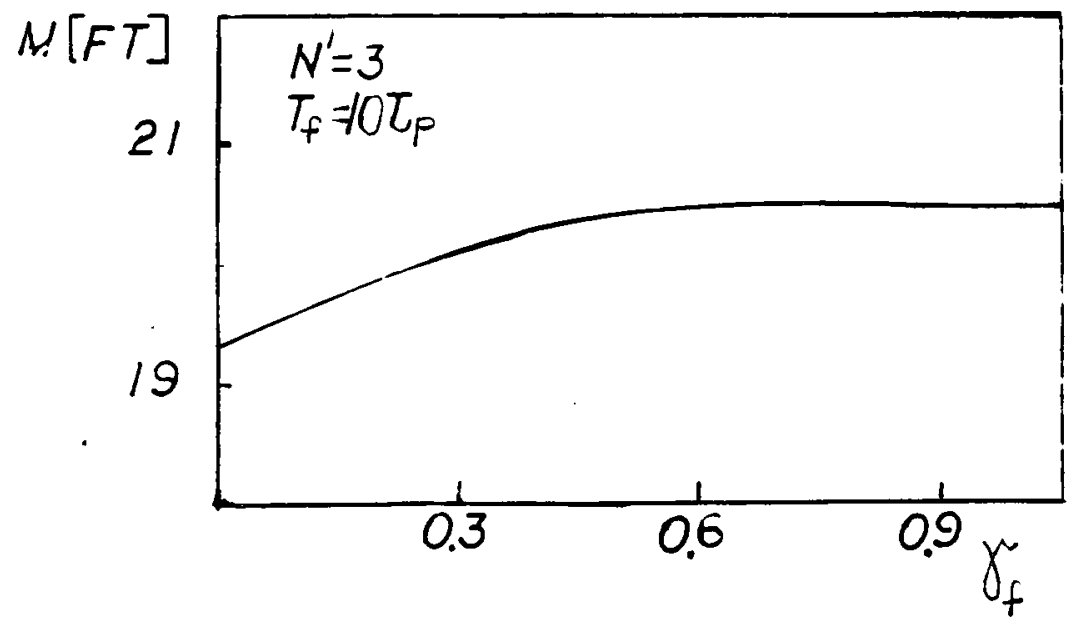

Figure 7: Miss Distance versus Terminal Path Angle for $t_{f}=10 \tau_{p}$ 


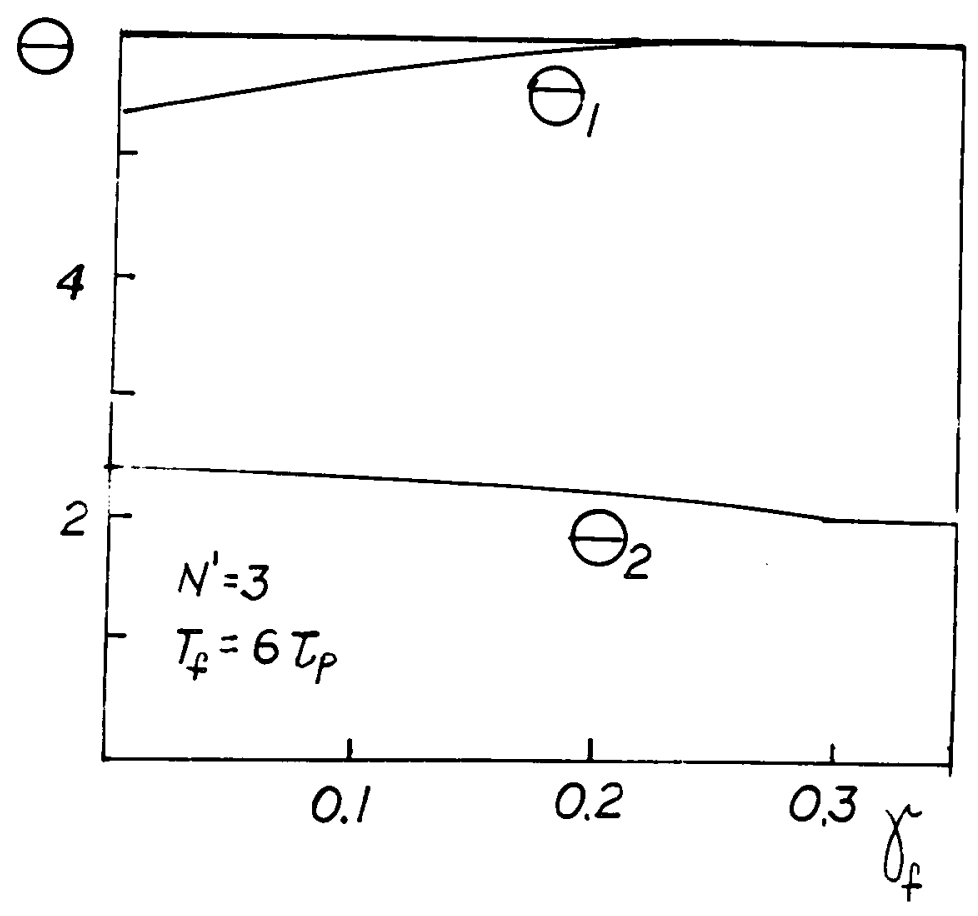

Figure 8: Switching Points versus Terminal Path Angle for $t_{f}=6 \tau_{p}$ 


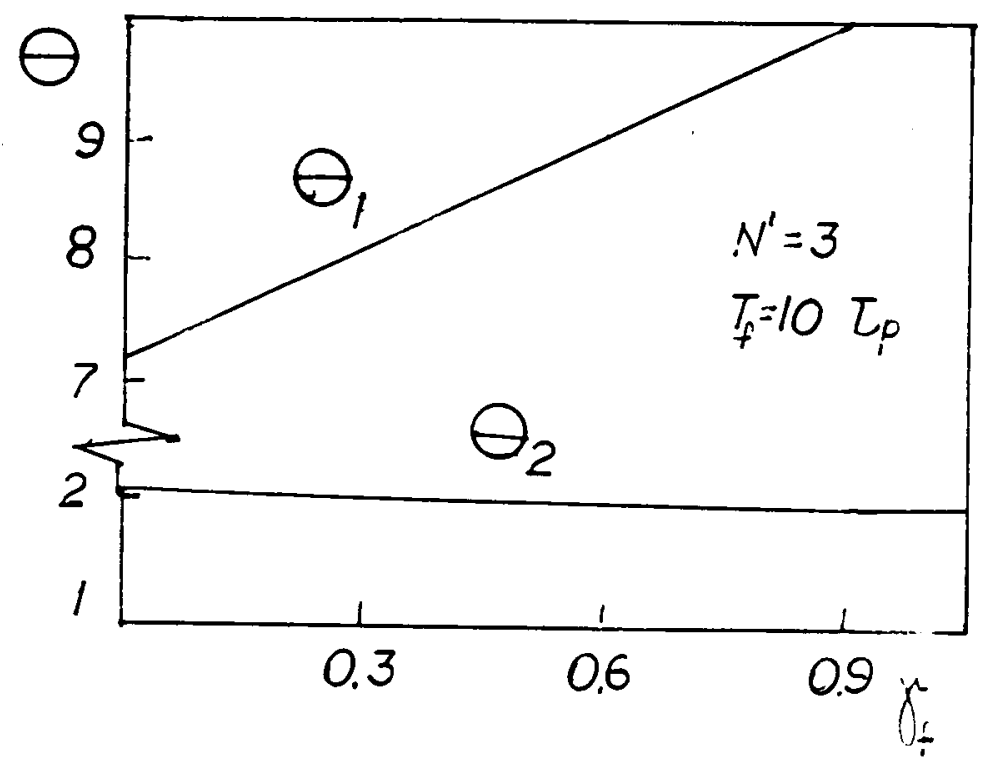

Figure 9: Switching Points versus Terminal Path Angle for $t_{f}=10 \tau_{p}$ 


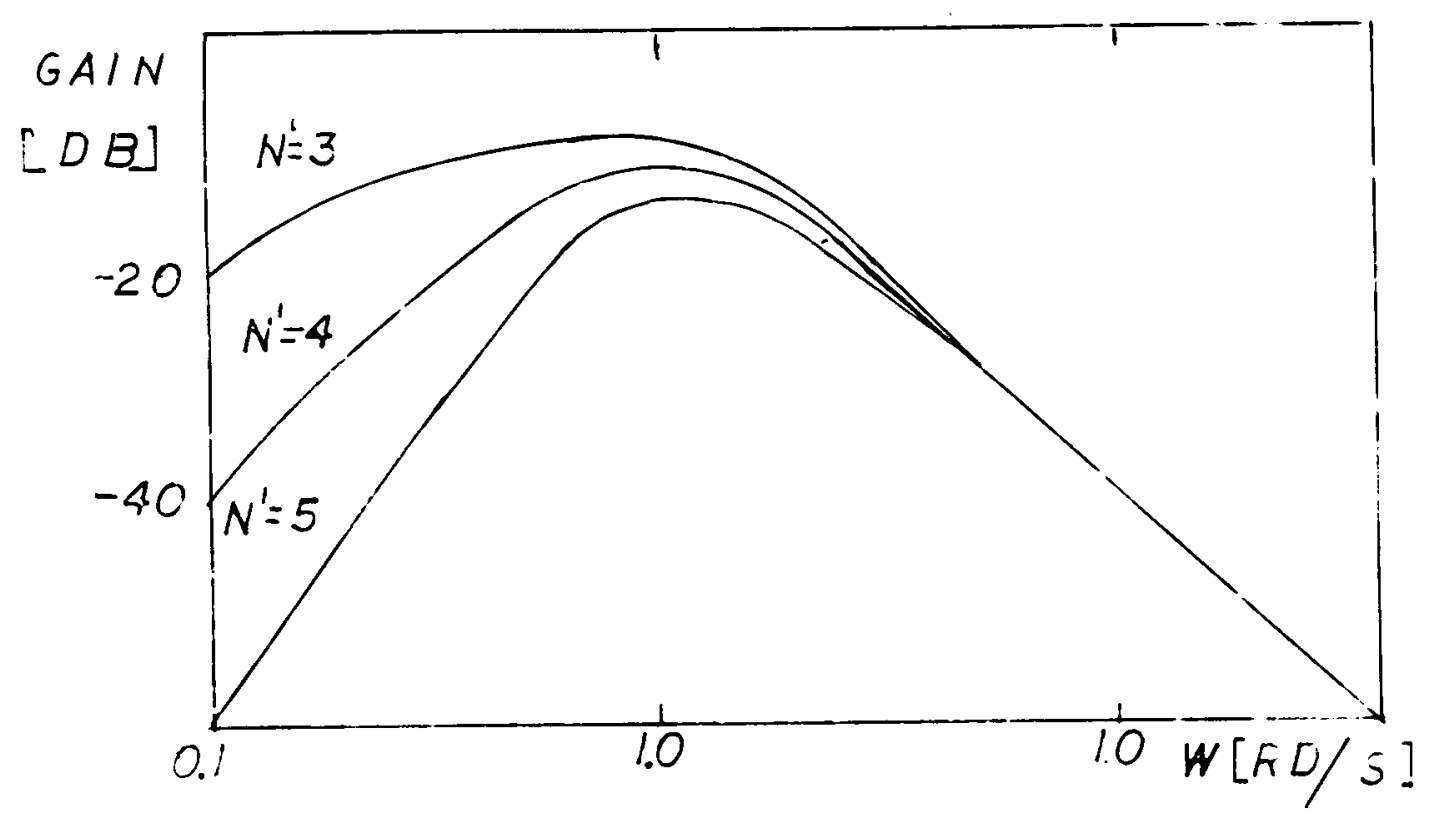

Figure 10 : Miss Distance Influence Function 


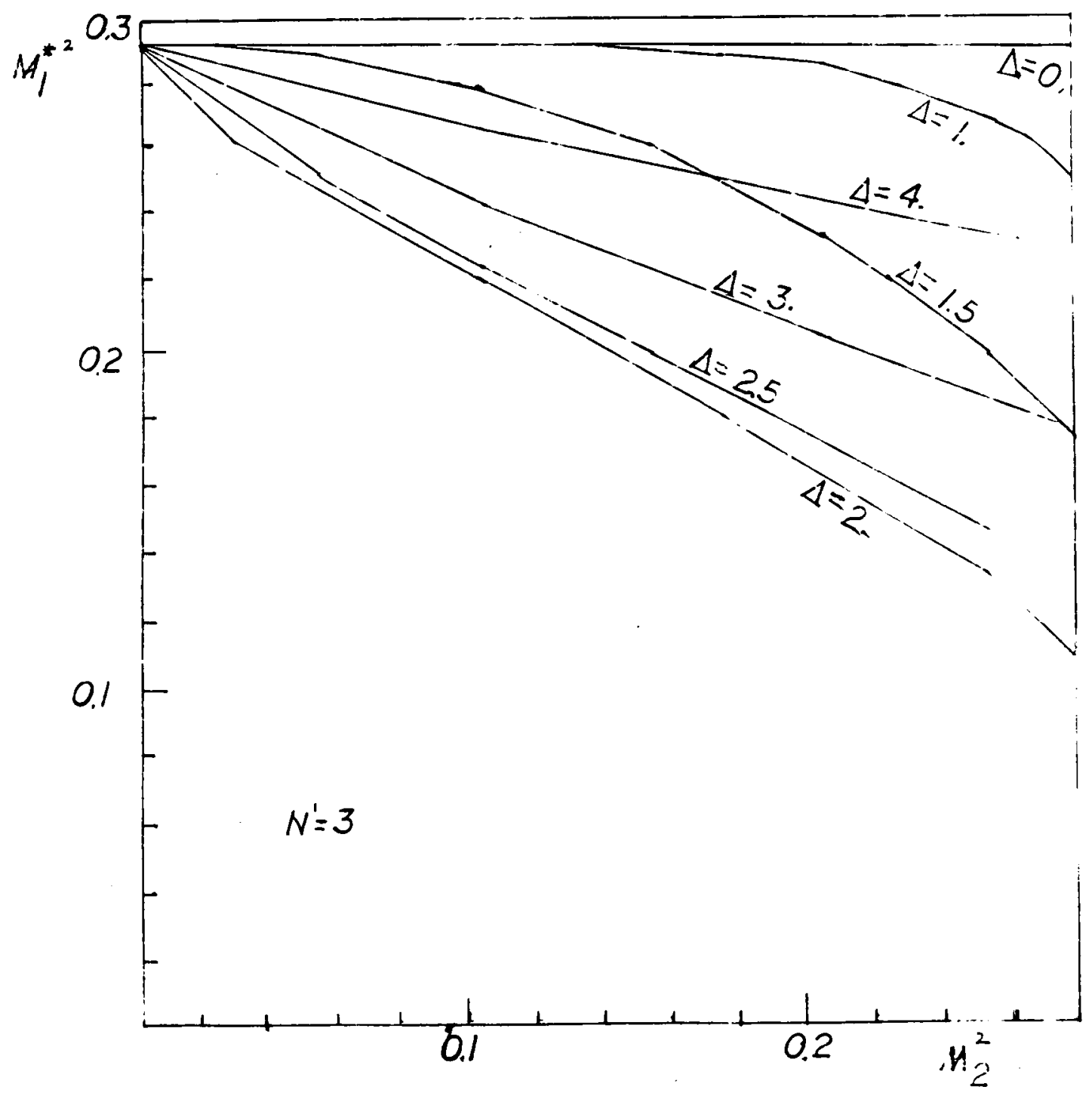

note: The miss distance is normalized by $V_{e} \tau_{p}^{2} \dot{\gamma}_{e_{m}}$

Figure 11 : Payoff versus Constraint for the Two Pusuer Problem 


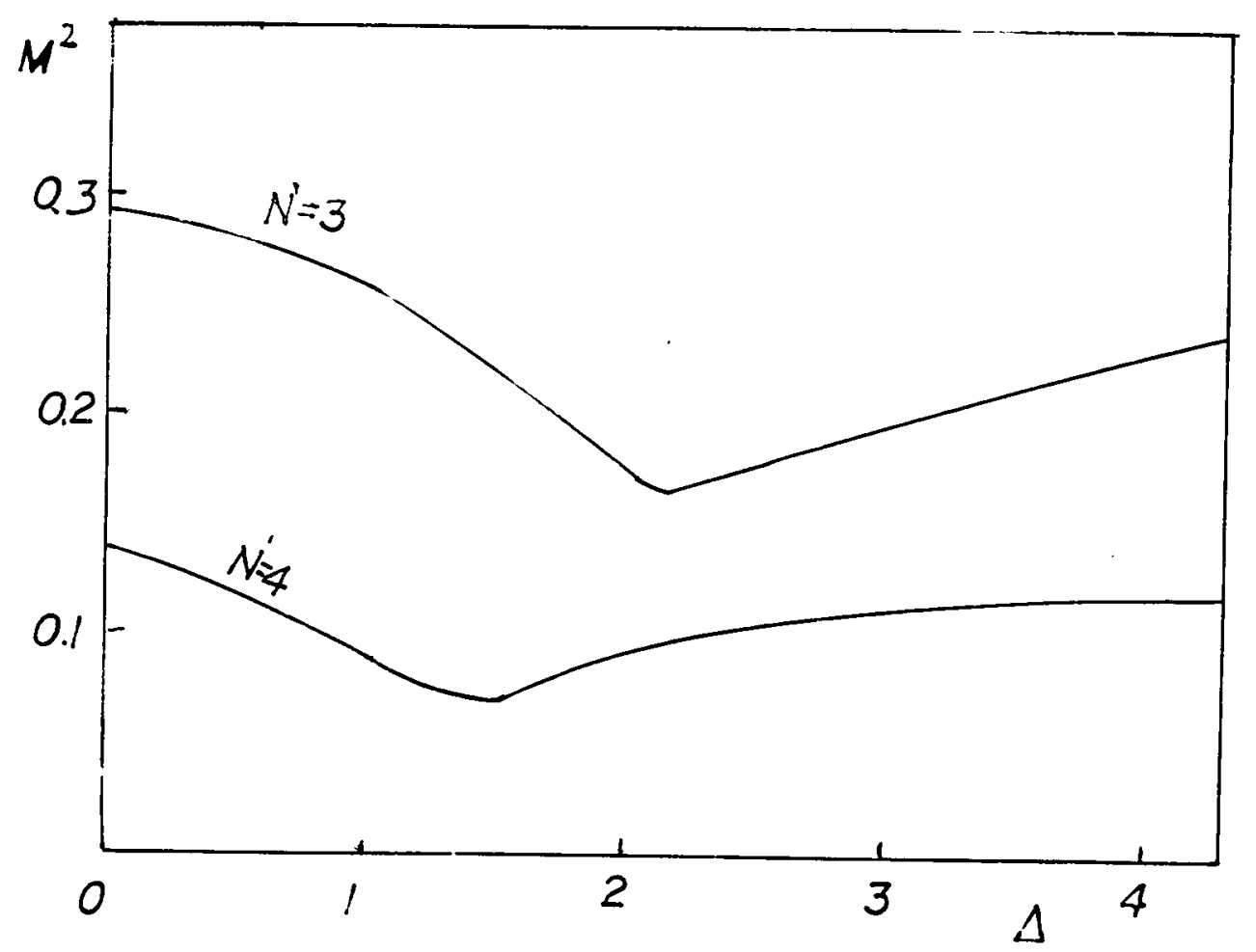

note: The miss distance is normalized by $V_{e} \tau_{p}^{2} \dot{\gamma}_{e_{m}}$

Figure 12 : Payoff versus Nominal Time Between Impacts for the Two Pursuer Problem. 


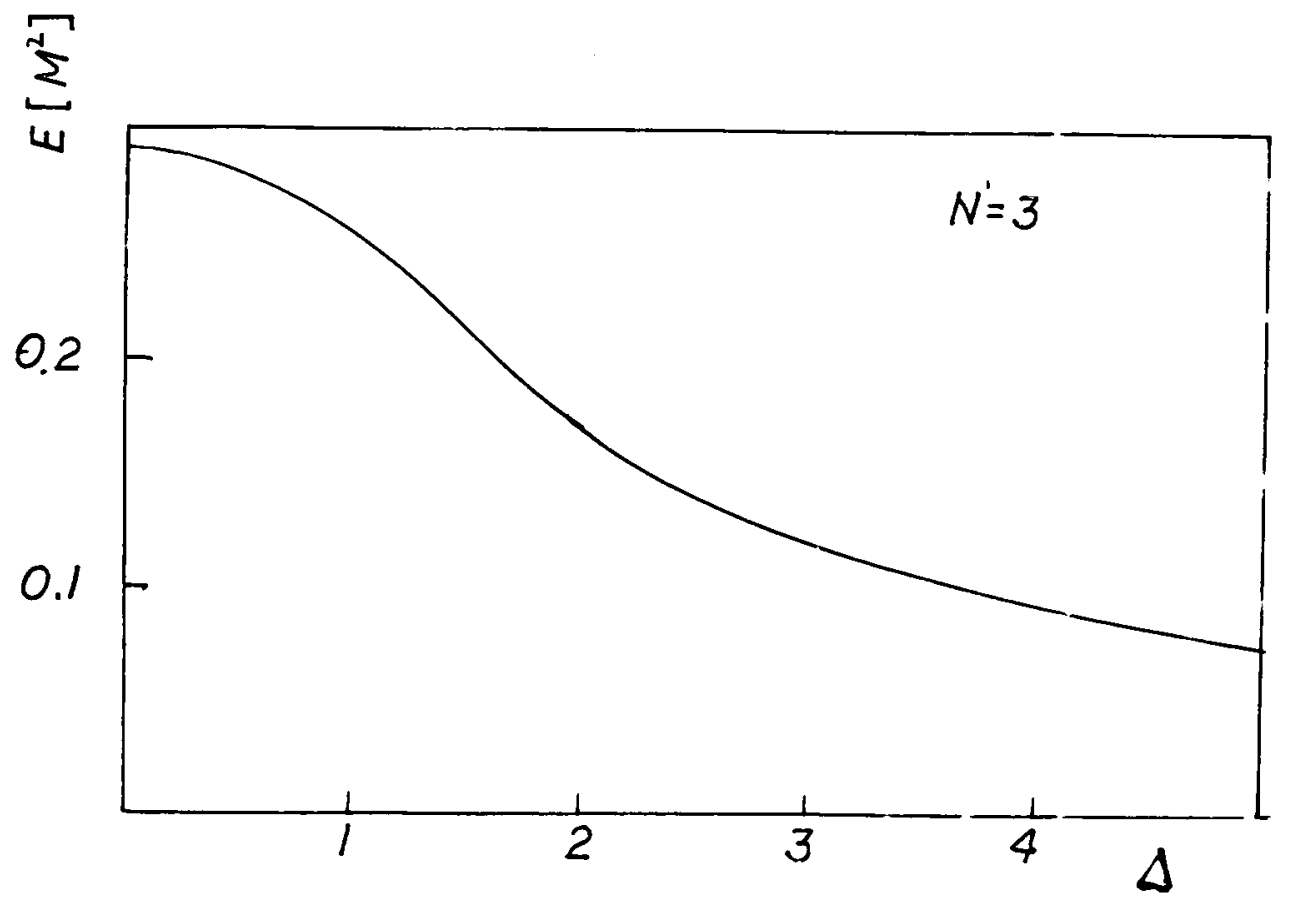

note : The miss distance is normalized by $V_{e} \tau_{p}^{2} \dot{\gamma}_{e_{m}}$

Figure 13: Expected Payoff versus Terminal Time Uncertainty 


\section{The vita has been removed from the scanned document}

